\title{
Quasiperiodic ULF-pulsations in Saturn's magnetosphere
}

\author{
G. Kleindienst ${ }^{1}$, K.-H. Glassmeier ${ }^{1,2}$, S. Simon ${ }^{3,}$, M. K. Dougherty ${ }^{4}$, and N. Krupp ${ }^{2}$ \\ ${ }^{1}$ Institut für Geophysik und extraterrestrische Physik, Technische Universität Braunschweig, Mendelssohnstraße 3, \\ 38106 Braunschweig, Germany \\ ${ }^{2}$ Max-Planck-Institut für Sonnensystemforschung, Max-Planck-Straße 2, 37191 Katlenburg-Lindau, Germany \\ ${ }^{3}$ Institut für Theoretische Physik, Technische Univ. Braunschweig, Mendelssohnstraße 3, 38106 Braunschweig, Germany \\ ${ }^{4}$ The Blackett Laboratory, Imperial College London, Prince Consort Road, London SW7 2BW, UK \\ * now at: Universität zu Köln, Institut für Geophysik und Meteorologie, Zülpicher Str. 49a, 50674 Köln, Germany
}

Received: 16 June 2008 - Revised: 18 December 2008 - Accepted: 27 January 2009 - Published: 23 February 2009

\begin{abstract}
Recent magnetic field investigations made onboard the Cassini spacecraft in the magnetosphere of Saturn show the existence of a variety of ultra low frequency plasma waves. Their frequencies suggest that they are presumably not eigenoscillations of the entire magnetospheric system, but excitations confined to selected regions of the magnetosphere. While the main magnetic field of Saturn shows a distinct large scale modulation of approximately $2 \mathrm{nT}$ with a periodicity close to Saturn's rotation period, these ULF pulsations are less obvious superimposed oscillations with an amplitude generally not larger than $3 \mathrm{nT}$ and show a packagelike structure. We have analyzed these wave packages and found that they are correlated to a certain extent with the large scale modulation of the main magnetic field. The spatial localization of the ULF wave activity is represented with respect to local time and Kronographic coordinates. For this purpose we introduce a method to correct the Kronographic longitude with respect to a rotation period different from its IAU definition. The observed wave packages occur in all magnetospheric regions independent of local time, elevation, or radial distance. Independent of the longitude correction applied the wave packages do not occur in an accentuated Kronographic longitude range, which implies that the waves are not excited or confined in the same selected longitude ranges at all times or that their lifetime leads to a variable phase with respect to the longitudes where they have been exited.
\end{abstract}

Keywords. Magnetospheric physics (Magnetospheric configuration and dynamics; MHD waves and instabilities; Planetary magnetospheres)

Correspondence to: G. Kleindienst (g.kleindienst@tu-bs.de)

\section{Introduction}

ULF waves are a widely observed phenomenon in various environments of the solar system. At comets pick-up of newlyionized particles leads to the generation of low-frequency waves at the local ion-gyro frequency (e.g. Tsurutani and Smith, 1986; Glassmeier and Neubauer, 1993; Glassmeier et al., 1993). The solar wind interaction regions of planets Venus and Mars are also prone to the generation of lowfrequency waves by pick-up ions (e.g. Luhmann et al., 1983; Espley et al., 2004). The terrestrial magnetosphere, however, is the classical environment to study ULF waves and eigenoscillations of an entire magnetosphere (e.g. Southwood and Hughes, 1983). Typical source mechanisms are the Kelvin-Helmholtz instability (e.g. Fujita et al., 1996), ringcurrent instabilities (e.g. Southwood, 1976), or solar wind buffeting (e.g. Mathie and Mann, 2000).

Apart from the well studied ULF waves in the Earth's magnetosphere, ULF waves can also be found in the magnetospheres of Mercury and the gaseous planets Jupiter and Saturn (e.g. Glassmeier et al., 2004). Khurana and Kivelson (1989) analyzed ULF waves confined in the equatorial region of the Jovian magnetosphere, while Glassmeier et al. (1989) reported about MHD waves confined to the Io plasma torus. Based on magnetic field investigations from Voyager 2, Cramm et al. (1998) showed evidence of ULF waves and resonant mode coupling in Saturn's magnetosphere.

The magnetospheres of Jupiter and Saturn are interesting environments to study MHD waves as the extensive size of these magnetospheric systems and rapid planetary rotation will most likely not support eigenoscillations of the entire magnetosphere. As the classical resonant coupling of compressional MHD waves to Alfvén modes in an inhomogeneous plasma environment, the so-called field-line resonance mechanism (e.g. Tamao, 1965; Southwood, 1974), implies

Published by Copernicus Publications on behalf of the European Geosciences Union. 

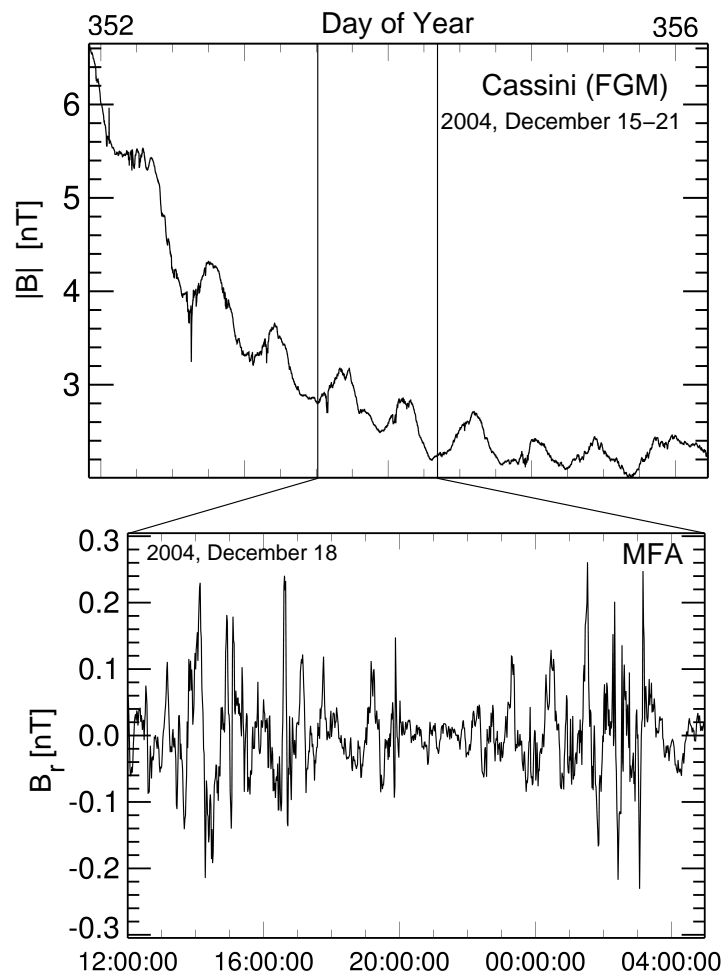

Fig. 1. Upper panel: magnitude of the main magnetic field over time (15-21 December 2004); bottom panel: the radial component of the magnetic field in a mean-field-aligned coordinate system, displaying two of the typical ULF waves packages centered around 18 December 2004, 15:00 UTC and 19 December 2004, 02:00 UTC.

the generation of standing toroidal magnetic field oscillations, the application of the field-line resonance theory to the Jovian and Kronian magnetosphere needs further analysis.

Recent studies based on the magnetic field investigation (Dougherty et al., 2004) of the Cassini mission into the Kronian system exhibit a variety of periodic magnetospheric perturbations at Saturn, amongst others a modulation of the magnetic field with a period close to the planetary rotation period (Giampieri et al., 2006), which has already been observed by the magnetometer experiments onboard the Voyager 1, Voyager 2 and Pioneer 11 spacecraft as a reanalysis of their magnetic field data demonstrates (Espinosa and Dougherty, 2000; Espinosa et al., 2003a). Giampieri et al. (2006) used this periodicity to derive a rotation period based on magnetic field measurements and yielded a period of $10 \mathrm{~h} 47 \mathrm{~min} 6 \mathrm{~s}$ which differs significantly from the rotation period of $10 \mathrm{~h} 39 \mathrm{~min} 22 \mathrm{~s}$ as officially defined by the IAU (Seidelmann et al., 2002) relying on earlier measurements by Desch and Kaiser (1981), based on the periodic occurrence of kilometric radiation in Saturn's high latitude regions.

The reported existence of magnetohydrodynamic waves in the magnetospheres of the gaseous planets as well as the initial findings of Espinosa et al. (2003b) and Giampieri et al.
(2006) motivated the present work. In the terrestrial magnetosphere several source mechanisms for ULF waves are known, while at Jupiter and Saturn knowledge about the wave source is still sparse.

Glassmeier (1995) suggested that the tilted and rotating Jovian dipole axis causes large-amplitude and periodic magnetic field perturbations in the Jovian magnetosphere whose non-linear decay is able to generate a variety of lowfrequency wave phenomena. As the magnetic dipole axis of Saturn is almost aligned with the rotation axis of the planet a direct extension of the suggestion of Glassmeier (1995) to the Kronian system is not possible. However, the large scale modulation of the Kronian magnetic field, whatever cause it has, can be regarded as a similar large-amplitude wave perturbation, which can be the source of ULF waves at Saturn. In the present study we thus concentrate on general wave properties and the spatial localization of the ULF waves in the Kronian magnetosphere and their relation to so far discovered magnetic features such as the periodic modulation of Saturn's main magnetic field. The frequency of the observed ULF pulsations indicates that they are not eigenoscillations of the entire Kronian magnetosphere, but are due to waves bound to specific regions of the magnetosphere. The identification of these regions is hampered by the definition of the Kronian longitude system (Seidelmann et al., 2002), which depends strongly on the accuracy of the planet's rotation period, which is just under discussion (Giampieri et al., 2006; Cecconi and Zarka, 2005; Gurnett et al., 2007; Goldreich and Farmer, 2007; Southwood and Kivelson, 2007). Thus, one of the aims of our study is also to find a suitable coordinate system, in which the observed ULF waves can best be represented, to understand their basic characteristics and to relate their occurrence to the global structure of the Kronian magnetosphere.

\section{ULF wave properties}

We analyse measurements from the vector fluxgate magnetometer of the Cassini spacecraft (Dougherty et al., 2004). For the present study we use magnetic field data with an averaged time resolution of $1 \mathrm{~min}$ along the path of the spacecraft's outbound trajectory from July 2004 to March 2007, inside the magnetosphere of Saturn, which corresponds to the first 40 revolutions of the spacecraft around the planet.

The spacecraft covered all local time ranges during this period of time and its orbits were close to the magnetic equator within a latitude range of $\pm 20^{\circ}$ for nearly $80 \%$ of the total time under consideration. Radial ranges from $3 R_{S}$ to $50 R_{S}$ were covered by the path of the satellite.

For our purpose of studying magnetohydrodynamic ULF waves a time resolution of $1 \mathrm{~min}$ and a corresponding Nyquist frequency of $8.3 \mathrm{mHz}$ is sufficient as the main plasma constituents of the Kronian magnetosphere $\left(\mathrm{O}^{+}\right.$, $\mathrm{OH}^{+}, \mathrm{O}_{2}^{+}, \mathrm{H}_{2} \mathrm{O}^{+}$, e.g. Ip (2000)) have typical cyclotron 
frequencies in the range of $5-10 \mathrm{mHz}$ for a background magnetic field of $10 \mathrm{nT}$. Thus, the observation of magnetohydrodynamic waves should be covered well enough by the chosen time resolution.

Figure 1 displays a typical example of the ULF waves analyzed. The oscillations are rather irregular and do usually not exhibit any quasi-sinusoidal character as often observed in the terrestrial magnetosphere. The wave activity is strongly modulated and resembles a certain correlation with variations of the background magnetic field. To access and distinguish these small amplitude oscillations from the background magnetic field and to distinguish oscillations transverse and parallel to the background magnetic field, we transform the magnetic field data into a Mean-Field-Aligned (MFA) coordinate system $\left(\boldsymbol{e}_{r}, \boldsymbol{e}_{\phi}, \boldsymbol{e}_{\|}\right)$, where $\boldsymbol{e}_{\|}$denotes the unit vector in the direction of the background magnetic field, $\boldsymbol{e}_{\phi}$ the unit vector perpendicular to the plane spanned by $\boldsymbol{e}_{\|}$and the spacecraft position vector $\boldsymbol{r}_{s c}$. The unit vector $\boldsymbol{e}_{r}$ completes the right hand $\left(\boldsymbol{e}_{r}, \boldsymbol{e}_{\phi}, \boldsymbol{e}_{\|}\right)$triad. The coordinate system used is thus defined as follows:

$\boldsymbol{e}_{r}=\boldsymbol{e}_{\phi} \times \boldsymbol{e}_{\|}$

$\boldsymbol{e}_{\phi}=\frac{\langle\boldsymbol{B}\rangle \times \boldsymbol{r}_{s c}}{\left|\langle\boldsymbol{B}\rangle \times \boldsymbol{r}_{s c}\right|}$

$\boldsymbol{e}_{\|}=\frac{\langle\boldsymbol{B}\rangle}{|\boldsymbol{B}|}$.

The mean magnetic field $\langle\boldsymbol{B}\rangle$ is determined by a second order polynominal fit within a sliding window of $141 \mathrm{~min}$ width, whose window size comprises the large scale modulation of the background magnetic field well. In the MFA-system $\left(b_{r}, b_{\phi}, b_{\|}\right)$denotes the magnetic disturbance field vectors. As we are only interested in field perturbations the mean value of the field-aligned component has been subtracted. The MFA-system allows one to inspect also the polarization of the analysed oscillations with $\left(b_{r}, b_{\phi}, b_{\|}\right)$approximately describing poloidal, toroidal, and field-aligned components, respectively.

A series of clearly distinguishable wave packages with mainly transverse amplitudes generally not larger than $0.5 \mathrm{nT}$ can be observed in the components perpendicular to the mean magnetic field for the chosen time interval. Figure 1 shows an interval of increased ULF wave activity as an example which exhibits two of the package-like structures centered around 18 December 2004, 15:00 UTC and 19 December 2004, 02:00 UTC, respectively.

The predominance of transverse over compressional fluctuations can be inferred from a spectral analysis of the two different components. Figure 2 shows a sample spectrum for the time interval 18 December 2004, 12:00 until 19 December 2004, 05:04 UT. The power spectral density distribution is rather featureless, does not exhibit any clear spectral peak being larger than the confidence limit, and decays with a spectral index of about $-5 / 3$. The perpendicular spectral density is at least an order of magnitude larger than the

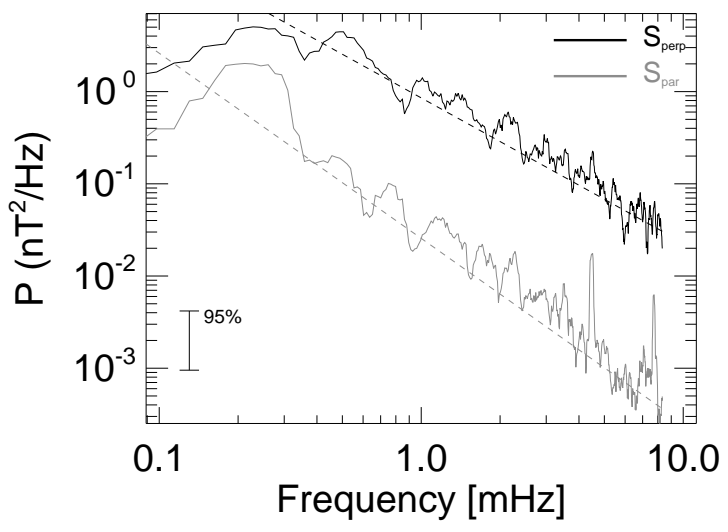

Fig. 2. Power spectral density of the ULF wave activity in the time interval 18 December 2004, 12:00 UTC to 19 December 2004, 05:04 UTC. The black line represents the sum of the spectral power density of the magnetic field fluctuations perpendicular to the background magnetic field. The grey line shows the spectral power density of the magnetic field fluctuations parallel to the background magnetic field. The dashed lines illustrate the spectral slopes fitted to each spectral power density.

parallel one. Therefore we classify the observed ULF wave activities as Alfvénic fluctuations.

The cross-spectral density which was calculated for the two transverse components $B_{r}$ and $B_{\phi}$ of the magnetic field represented in Fig. 3 shows, that no maximum is clearly accentuated from the background spectrum and the coherency has values generally much smaller then $\gamma=0.7$, which implies that the degree of polarization in the plane perpendicular to the background magnetic field is also small. Before investigating this correlation any further we like to comment on any wave activity distribution as observed in the Kronian magnetosphere.

Because of the rather irregular level of fluctuations we use the Carson-Gabor or analytical signal representation (Glassmeier, 1980) to yield an instantaneous amplitude of the magnetic field oscillations in order to determine and calculate a wave activity index. In this representation $z(t)=x(t)+i y(t)$ is a complex time series with $x(t)$ representing the original time series and $y(t)$ being defined as

$y(t)=\mathcal{H}(x(t))$,

where $\mathcal{H}$ denotes the Hilbert-transform. The instantaneous amplitude $A(t)$ is now given as

$A(t)=\sqrt{x^{2}(t)+y^{2}(t)}$.

We define a wave activity index $W(t)$ for each magnetic field component as the hourly time average of the instantaneous amplitude. As an example Fig. 4 displays the radial variation of the transverse wave index, $W_{\perp}=\left(W_{r}+W_{\phi}\right) / 2$, together with the magnitude of the background magnetic field as a 


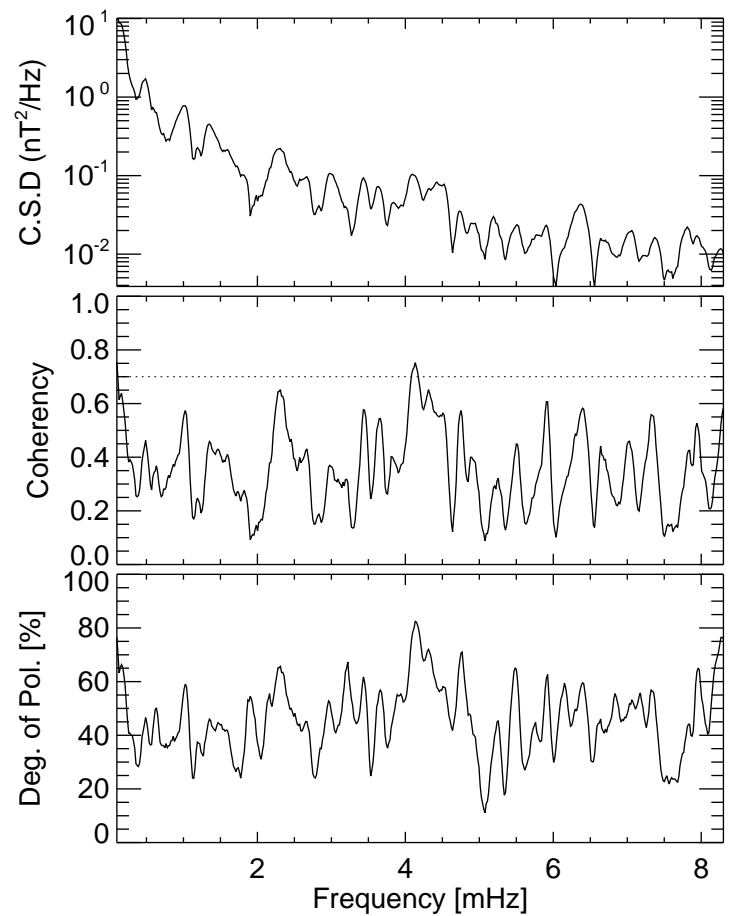

Fig. 3. The cross spectral density of the ULF wave packages in the time interval from 18 December 2004, 12:00 UTC and 19 December 2004, 05:04 UTC for the two transverse magnetic field components.

function of radial distance for the time interval 15 December 2004-22 December 2004; indices denote the magnetic field components used. Apparently the wave activity correlates with the large scale variation of the magnetic field strength. To quantify this possible correlation we formally determine the cross correlation function between both time series. To remove the trend of the total magnetic field we have fitted a dipolar declining function to the minima of the total field radial variation. The resulting residuum is correlated with the wave activity index for a maximum lag of $1500 \mathrm{~min}$. The first local maximum occurs for a lag time of approximately $-2 \mathrm{~h}$, that is the background magnetic field reaches its maximum two hours after the wave activity maximizes. The crosscorrelation function shows a clear periodic structure with a maximum correlation coefficient of about 0.5 . This correlation coefficient is lower than anticipated by visual inspection of Fig. 4 and due to variations of the instantaneous amplitude at around $24 R_{S}$ and $37 R_{S}$. Correlating the two time series between $26 R_{S}$ and $35 R_{S}$ gives one a maximum correlation coefficient of 0.75 and a corresponding lag time of $-98 \mathrm{~min}$ (Fig. 5). We conclude that there is an interesting relation between ULF wave activity and the variation of the background magnetic field.

To further analyze the wave activity distribution we use the data in the MFA-system to calculate the total wave activity index $W_{\text {tot }}=\frac{1}{3}\left(W_{r}+W_{\phi}+W_{\|}\right)$, the transverse wave activity

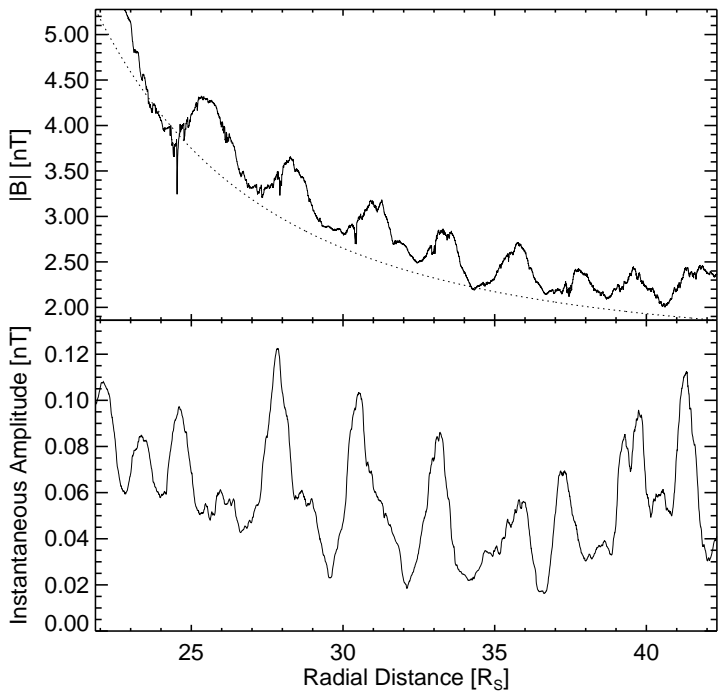

Fig. 4. Upper panel: magnitude of the main magnetic field over radial distance in the time interval from 17 December 2004, 06:00 UTC and 21 December 2004, 03:00 UTC. The dashed line shows the trend which has be subtracted from the magnetic field magnitude for further correlation analysis. Bottom panel: transverse wave activity index $W_{\perp}$ over radial distance.

index $W_{\perp}$, and the parallel wave activity index $W_{\|}$for those time intervals, where the spacecraft was inside Saturn's magnetosphere. Wave activity distribution functions are determined using a bin size of $0.1 \mathrm{nT}$. The resulting distributions in Fig. 6 indicate, that for all three indices the wave activity index declines exponentially for larger indices. The total wave index distribution shows that most fluctuations have small amplitudes, generally not larger than $3 \mathrm{nT}$, which are all situated well above the instrumental noise-level which is stated to be better than $25 \mathrm{pT}^{2} / \mathrm{Hz}$ (Dougherty et al., 2004).

The transverse and compressible distributions bear a similar feature. For wave indices less than $0.5 \mathrm{nT}$ we observe a higher number of transverse wave indices as compared to parallel wave indices, while for larger amplitudes up to $1.5 \mathrm{nT}$ both polarizations contribute equally. For amplitudes larger than $1.5 \mathrm{nT}$ the number of parallel wave indices are slightly larger compared to the transverse wave indices.

To investigate a possible dependence of wave activity on the magnetospheric regions they have been detected in, we calculated distributions of the total wave activity index with respect to magnetic local time, radial distance, and magnetic elevation.

Total wave activity is clearly enhanced between $3-6 \mathrm{~h}$ and around $23 \mathrm{~h}$ magnetic local time. However, to exclude any dependence on the probability of the Cassini spacecraft visiting a certain region of the magnetosphere the distributions need to be normalized to the corresponding distribution of the probability to find the spacecraft in a specified region. Figure 7 shows the propability to find the spacecraft in each 


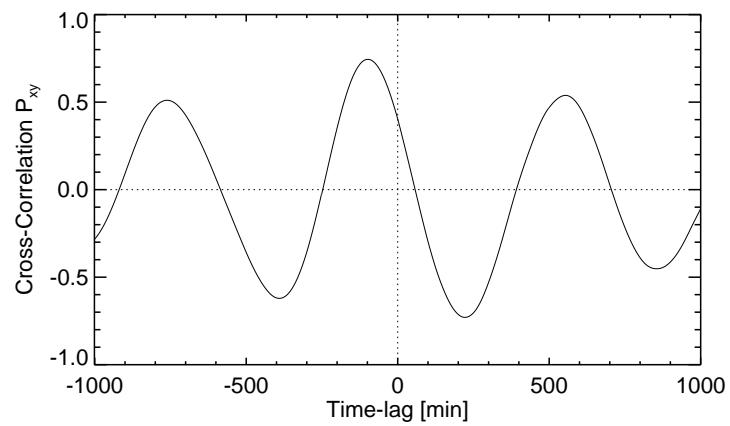

Fig. 5. Cross-Correlation of the detrended magnitude of the magnetic field and the wave activity index for the subinterval of the time series depicted in Fig. 4 restricted to the the radial range between $26 R_{S}$ and $35 R_{S}$. The first maximum peaks at a time-lag of approx. $100 \mathrm{~min}$. A negative time-lag implies that the detrended magnitude of the magnetic field has to be shifted backwards in time to show a maximum correlation with the instantaneous amplitude of the wave activity index, thus appearing along the rising flanks of the magnetic magnitude.

local time region with respect to a binsize of $1 \mathrm{~h}$. Such a normalized distribution (Fig. 8) exhibits that wave activity indices are generally not larger than $1 \mathrm{nT}$ and more or less uniformly distributed over magnetic local times. There is a slight indication for larger amplitudes of about $0.5 \mathrm{nT}$ around local noon. However we do not regard this as a significant structure and would like to conclude a rather equal local time distribution from the observed distribution. In a similar way we also determine normalized distributions as a function of radial distance and magnetic elevation. The radial distribution is almost uniform over all radial distances with wave activity indices generally not larger than $0.5 \mathrm{nT}$. The magnetic elevation dependent normalized distribution indicates only a minor enhancement for magnetic elevations larger than $\pm 30^{\circ}$ and is uniform otherwise. The minor enhancement is most probably an artifact, as the spacecraft's probability distribution is fairly low for these elevations and thus the normalized distribution is over-emphasized for elevations beyond $\pm 30^{\circ}$. We thus conclude that ULF wave activity of comparable magnitude is observed all over the magnetospheric regions visited by Cassini so far.

\section{ULF wave activity and a rotation-related coordinate system}

The relation between background magnetic field and ULF wave activity suggested by the results presented in Figs. 4 and 5 deserves further attention as we found similar correlations also for other time intervals.

To further our hypotheses it is desirable to represent the wave activity in a rotation-related coordinate system. The spacecraft's position with respect to standard Kronographic

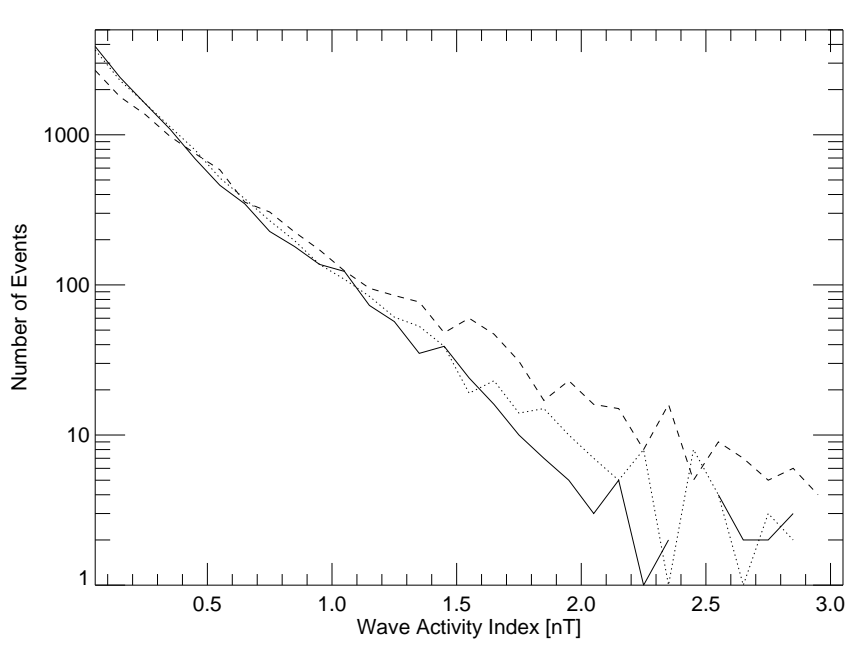

Fig. 6. Wave activity index distributions: the dashed lines gives the distribution of the total wave activity index, the solid one that of the tranverse oscillations, and the dotted line denotes the compressible perturbation index. The one hour time average of the wave activity index is counted as one event in the statistic.

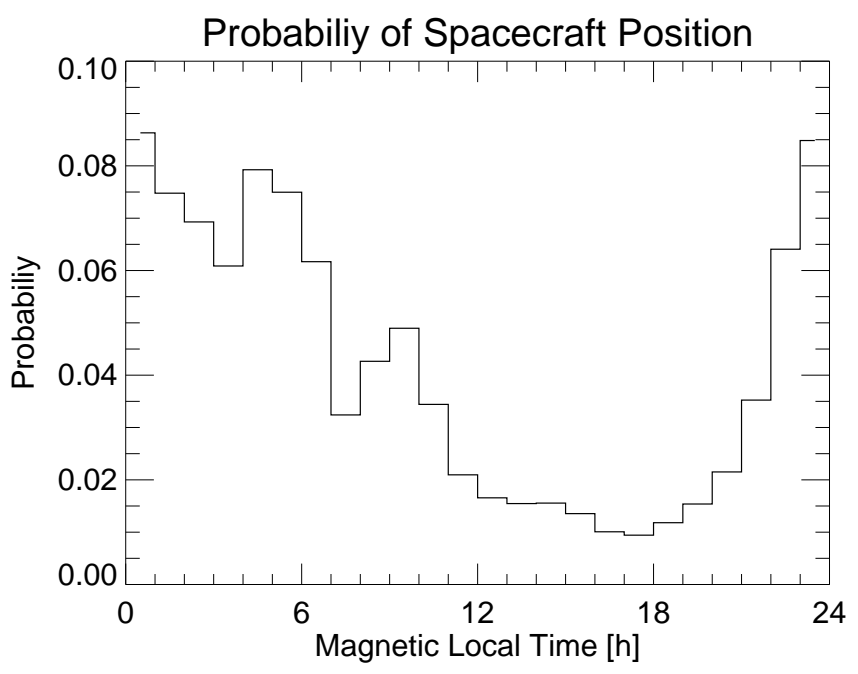

Fig. 7. Histogram of the probability to find the spacecraft within a magnetic local time range, which is used to normalize the distribution of the hourly averaged total wave activity index with respect to their amplitude and magnetic local time.

coordinates is calculated by means of JPL's Navigation and Ancillary Information Facility (NAIF) Toolkit (Acton, 1999). Saturn's ephemerides used by the NAIF toolkit are based on the rotation period defined by the IAU (Seidelmann et al., 2002). However, a time-variable longitude system needs to be introduced as studies of (Giampieri et al., 2006), using the periodic modulation of Saturn's main magnetic field, derived a rotation period of $10 \mathrm{~h} 47 \mathrm{~min} 6 \mathrm{~s}$ differing significantly from the officially defined rotation period of $10 \mathrm{~h} 39 \mathrm{~min} 22 \mathrm{~s}$ 


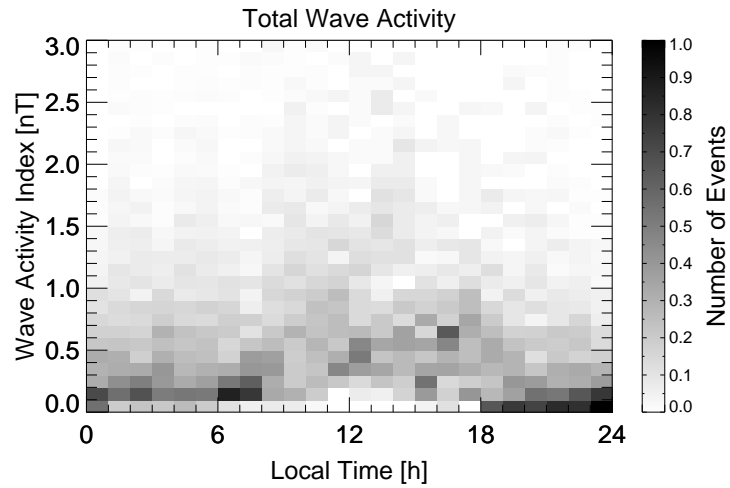

Fig. 8. The histogram shows the number of wave events of the hourly averaged total wave activity index with respect to their amplitude and magnetic local time distribution. The local time distribution has been normalized with the probability distribution of the spacecraft's local time position. The resulting histogram is normalized to one. The histogram does not show any region of pronounced wave activity and the number of wave indices is more uniformly distributed then before the normalization. Only a slight indication for an enhanced wave activity with marginal larger amplitudes may be observed around local noon.

given by the IAU (Seidelmann et al., 2002). This difference between the IAU and Giampieri et al. (2006) rotation periods indicates a variable apparent Kronian rotation rate. As a consequence Giampieri et al. (2006) suggested to correct the Saturn prime meridian and the rotation period accordingly. Any localization of physical processes such as the observed wave activity with respect to Kronian longitude will become obsolete if an incorrect rotation rate and longitude system is used. Therefore we need to correct the longitude of any wave activity observation with respect to the new, magnetic field based rotation period. The simplest method to introduce the required correction is to consider the phase shift of the prime meridian arising from the difference between $T_{\mathrm{IAU}}=10 \mathrm{~h} 39 \min 22 \mathrm{~s}$ and $T_{\mathrm{Cassini}}=10 \mathrm{~h} 47 \min 6 \mathrm{~s}$. If the longitude of the prime meridian at the beginning of the $J 2000$ epoch ( 1 January 2000, 12:00:00 UT) is $\varphi_{0}=38.90^{\circ}$ (Seidelmann et al., 2002), the phase evolution due to the two differing rotation periods, $T_{\text {Cassini }}$ and $T_{\mathrm{IAU}}$, can be calculated. In the following the rotation rate $\omega=360^{\circ} / T(\% / s)$ is used for further considerations. We first consider the phase evolution of the prime meridian due to a constant defined rotation rate $\omega_{\mathrm{IAU}}$ and due to a variable rotation rate $\omega_{\mathrm{Krono}}(t)$ :

$$
\begin{aligned}
\varphi_{\mathrm{IAU}}(t) & =\varphi_{0}\left(t_{0}\right)+\omega_{\mathrm{IAU}}\left(t-t_{0}\right) \\
\varphi_{\mathrm{Krono}}(t) & =\varphi_{0}\left(t_{0}\right)+\int_{t_{0}}^{t} \omega_{\mathrm{Krono}}(\tau) \mathrm{d} \tau
\end{aligned}
$$

where $t$ is the time elapsed since $t_{0}$. Obviously the prime meridian has a different time evolution for the two rotation rates and the phase difference between them can be expressed as

$$
\begin{aligned}
\varphi_{\mathrm{Krono}}(t) & =\varphi_{\mathrm{IAU}}(t)+\Delta \varphi(t) \\
\Delta \varphi(t) & =\varphi_{\mathrm{Krono}}(t)-\varphi_{\mathrm{IAU}}(t) \\
& =\int_{t_{0}}^{t} \omega_{\mathrm{Krono}}(\tau) \mathrm{d} \tau-\omega_{\mathrm{IAU}}\left(t-t_{0}\right) .
\end{aligned}
$$

Any Kronographic longitude $\lambda_{\text {IAU }}$ calculated on the basis of $\omega_{\text {IAU }}$ needs to be corrected with respect to this phase difference $\Delta \varphi(t)$ in order to obtain a modified longitude $\lambda_{\text {Krono }}$ which is stationary with respect to the prime meridian at $\varphi_{\text {Krono }}(t)$. The corrected longitude in the modified Kronographic coordinate system thus reads:

$$
\begin{aligned}
& \lambda_{\mathrm{Krono}}(t)=\lambda_{\mathrm{IAU}}(t)+\Delta \varphi(t) \\
& \lambda_{\mathrm{Krono}}(t)=\lambda_{\mathrm{IAU}}(t)-\omega_{\mathrm{IAU}}\left(t-t_{0}\right)+\int_{t_{0}}^{t} \omega_{\mathrm{Krono}}(\tau) \mathrm{d} \tau
\end{aligned}
$$

If one assumes that $\omega_{\text {Krono }}$ is constant in time and the prime meridians were congruent at $t_{0}$, the phase shift between a prime meridian calculated with respect to $\omega_{\mathrm{IAU}}=9.384 \times 10^{-30} / \mathrm{s} \quad\left(T_{\mathrm{IAU}}=10 \mathrm{~h} 39 \mathrm{~min} 22 \mathrm{~s}\right)$ and $\omega_{\text {Cassini }}=9.272 \times 10^{-30} / \mathrm{s}\left(T_{\text {Cassini }}=10 \mathrm{~h} 47 \mathrm{~min} 6 \mathrm{~s}\right)$ is

$$
\begin{aligned}
\Delta \varphi(t) & =\varphi_{\mathrm{Krono}}(t)-\varphi_{\mathrm{IAU}}(t) \\
& =\omega_{\mathrm{Krono}}\left(t-t_{0}\right)-\omega_{\mathrm{IAU}}\left(t-t_{0}\right) \\
& =\underbrace{\left(\omega_{\mathrm{Krono}}-\omega_{\mathrm{IAU}}\right)}_{\Delta \omega}\left(t-t_{0}\right) .
\end{aligned}
$$

with $\Delta \omega=-0.1127 \times 10^{-30} / \mathrm{s}(\Delta T=7 \mathrm{~min} 44 \mathrm{~s})$. This leads to a phase shift of the prime meridian of $4.3^{\circ}$ per nominal rotation and sums up to a complete rotation every 37.2 days. The longitude determined with any standard tool such as the NAIF Toolkit is easily corrected by adding the phase shift of the prime meridian:

$\lambda_{\mathrm{Krono}}(t)=\lambda_{\mathrm{IAU}}(t)+\Delta \varphi(t)$

Longitudes corrected in this way are used to infer any localization of the observed ULF waves in a Kronographic coordinate system.

Above considerations can also be used to determine the actual rotation period by observing a certain physical feature and its dependence on longitude. To illustrate this let us consider a stationary structure on Saturn's surface, e.g. an anomaly as suggested by Galopeau et al. (1991), which causes a magnetic signal measurable at the position of the spacecraft. An observer, who calculates the longitude with the correct actual rotation rate will measure this signal always at the same longitude, while an observer who uses an ambiguous rotation rate for his longitude calculation will observe a longitudinal drift motion of the signal's source, resulting in a dispersed distribution of the signal with longitude. From the observed drift motion of a signal $S$ one can calculate the correct actual rotation rate by using the relation 


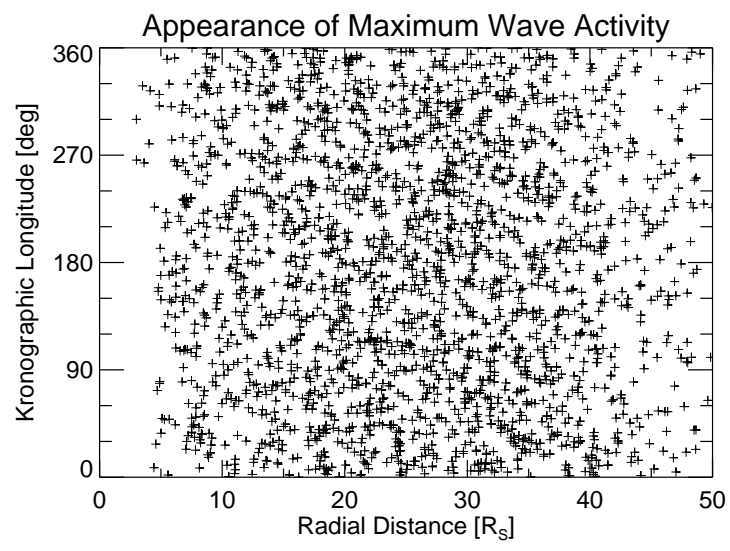

Fig. 9. Position of the maxima of the wave activity index in kronographic longitude over radial distance based on the rotation period of $10 \mathrm{~h} 39 \mathrm{~min} 22 \mathrm{~s}$ defined by the IAU (Seidelmann et al., 2002).

$$
\begin{aligned}
\lambda_{\mathrm{S}, \mathrm{Krono}}(t) & =\lambda_{\mathrm{S}, \mathrm{IAU}}(t)+\Delta \varphi(t) \\
& =\lambda_{\mathrm{S}, \mathrm{IAU}}(t)+\left(\int_{t_{0}}^{t} \omega_{\mathrm{Krono}}(\tau) \mathrm{d} \tau-\omega_{\mathrm{IAU}}\left(t-t_{0}\right)\right) .
\end{aligned}
$$

From this the time derivative of $\lambda_{\mathrm{S}, \mathrm{IAU}}(t)$ as well as the true rotation period can be determined, because $\mathrm{d} \lambda_{\mathrm{S}, \mathrm{Krono}} / \mathrm{dt}=0$ for a stationary source:

$$
\begin{aligned}
\frac{\mathrm{d} \lambda_{\mathrm{S}, \mathrm{IAU}}(t)}{\mathrm{dt}} & =-\left(\omega_{\mathrm{Krono}}(t)-\omega_{\mathrm{IAU}}\right) \\
\omega_{\mathrm{Krono}}(t) & =\omega_{\mathrm{IAU}}-\frac{\mathrm{d} \lambda_{\mathrm{S}, \mathrm{IAU}}(t)}{\mathrm{dt}} .
\end{aligned}
$$

The above considerations are used to study any possible localization of the wave activity in the Kronian magnetosphere and its relation to the modulation of the background magnetic field. For the time interval July 2004-January 2007 the maxima of the activity index are shown as a function of the standard Kronographic longitude $\lambda$ and radial distance $R$ from the planet (Fig. 9). A total number of 1630 maxima of the total wave activity index have been selected by an automatic algorithm.

No structured distribution is observed, but the wave activity maxima are uniformly scattered in longitude and radial distance. Correcting the longitude as discussed above and using the rotation period suggested by Giampieri et al. (2006) yields a slightly different distribution (not shown here), but still an almost uniform one. This either implies that the source of the wave activity is not stationary, or it hints towards a variable rotation rate. To check this later possibility we derive a variable rotation rate based on the modulation of the z-component of the background magnetic field, which best exhibits the periodic variations of the magnetic

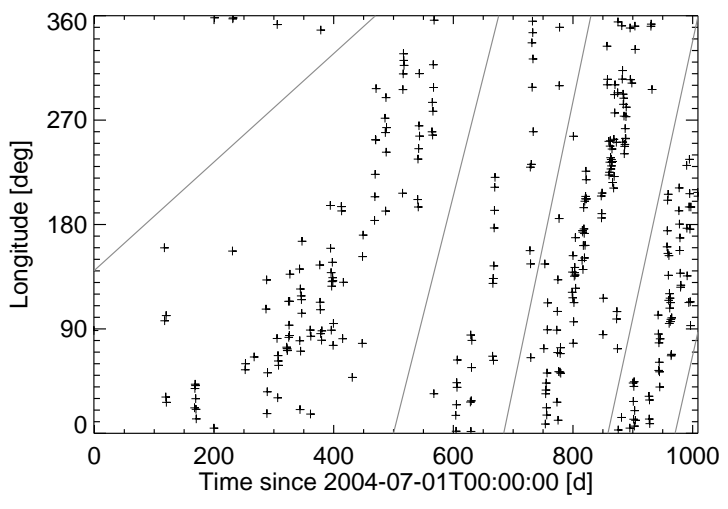

Fig. 10. Longitude representation of the maxima of the zcomponent of the magnetic field modulation based on a rotation period of $10 \mathrm{~h} 47 \mathrm{~min} 6 \mathrm{~s}$ according to the results of Giampieri et al. (2006). The grey lines mark the regions to be shifted by an integer multiple of $360^{\circ}$ to produce the continous longitude representation of the displayed maxima. The time axis starts on day 2004-148.

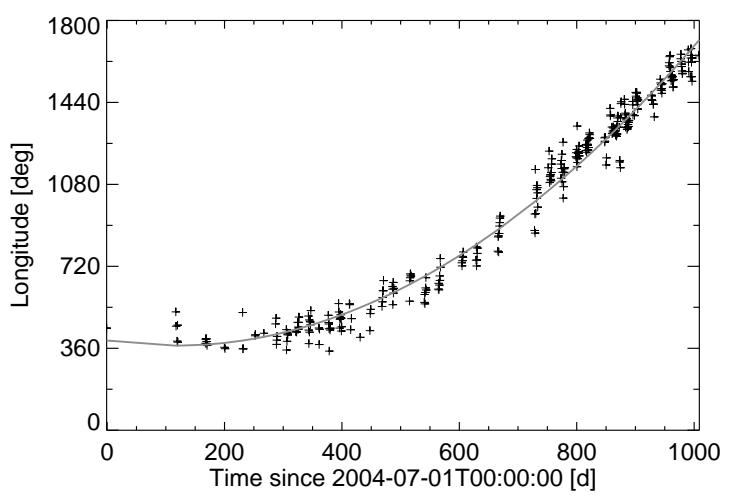

Fig. 11. Longitude representation of the maxima of the $\mathrm{z}$ component of the magnetic field modulation based on a rotation period of $10 \mathrm{~h} 47 \mathrm{~min} 6 \mathrm{~s}$ according to the results of Giampieri et al. (2006) with data points shifted by $n \cdot 360^{\circ}, n \in\{0,1,2, \ldots\}$ in the selected regions divided by the grey lines in Fig. 10 to obtain a continuous representation of the maxima without phase jumps at $360^{\circ}$. The line represents the best fit model for a polynominal function to describe the drift-curve due to an erroneous rotation period. The time axis starts on day 2004-148.

field. Figure 10 displays the maxima of the z-component as a function of longitude and time of occurrence. Corrected longitude based on the Giampieri et al. (2006) rotation period is used. A structured pattern with clearly visible bands of $\mathrm{z}$ component maxima can be identified. It is suggestive to view these bands as $360^{\circ}$ phase skipped parts of one and the same longitude function. Correcting for these phase jumps gives one the longitude-time curve displayed in Fig. 11. The continuous longitude curve resulting in this way either indicates that the source region of the background field modulation is 


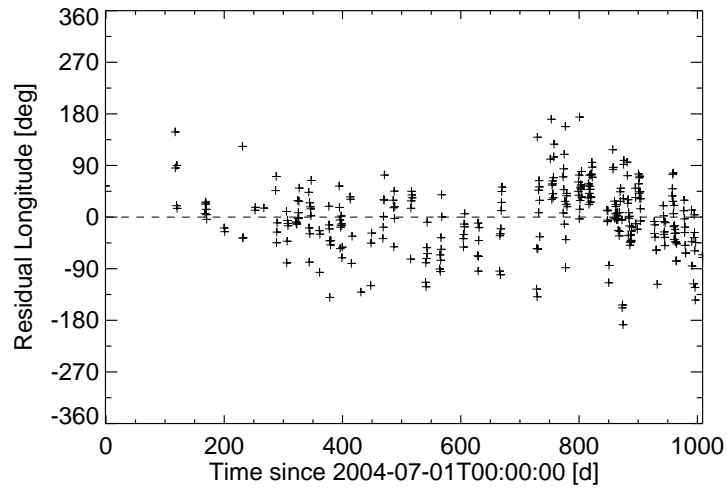

Fig. 12. Longitude representation of the maxima of the $\mathrm{z}$ component of the magnetic field modulation after removal of the fitted driftcurve to the datapoints, thus correcting the observed longitudes with respect to a time-varying rotation period. The time axis starts on day 2004-148.

drifting in longitude or a still incorrect rotation rate is used. If this latter hypothesis is correct a time-varying rotation period needs to be assumed. To determine the apparent temporal variation of the rotation frequency we have fitted a quadratic function of the form

$\lambda_{B_{z, \max }}(t)=\lambda_{0}+\alpha \cdot t+\beta \cdot\left(t-t_{0}\right)^{2}$

to the curve displayed in Fig. 11. The following parameters are found:

$\lambda_{0}=(34.09 \pm 15.3)^{\circ}$

$\alpha=(1.37 \pm 1.70) \times 10^{-5} \% / \mathrm{s}$

$\beta=(2.283 \pm 0.077) \times 10^{-13 \circ} / \mathrm{s}^{2}$

$t_{0}=(40622810 \pm 373500) \mathrm{s} \approx(470 \pm 4.3) \mathrm{d}$

where the time $t$ is counted in s since 1 July 2004, 00:00 UT. With

$$
\begin{aligned}
\omega_{\text {Krono }}(t) & =\omega_{\text {Cassini }}-\frac{\mathrm{d} \lambda_{B_{z, \max }}(t)}{\mathrm{dt}} \\
& =\omega_{\text {Cassini }}-\left(\alpha+2 \beta\left(t-t_{0}\right)\right)
\end{aligned}
$$

we obtain a rotation rate

$9.278 \times 10^{-5 \circ} / \mathrm{s}>\omega_{\text {Krono }}(t)>9.238 \times 10^{-5 \circ} / \mathrm{s}$

$\left(38800 \mathrm{~s}<T_{\text {Krono }}<38970 \mathrm{~s}\right)$ for the beginning (1 July 2004) and the end (30 January 2007) of the considered time interval for the longitude drift.

With this variable rotation rate we can also correct the longitude-time distribution of the background magnetic modulation (Fig. 12). Though considerable scattering is still apparent, the putative source location of the disturber causing the background magnetic field modulation is well constrained. The time-varying rotation rate and period function can also be extrapolated to past times to enable a comparison with earlier measurements of the rotation rate by e.g. Voyager 1 . For the year 1980 a rotation period of $T=37366 \mathrm{~s}$

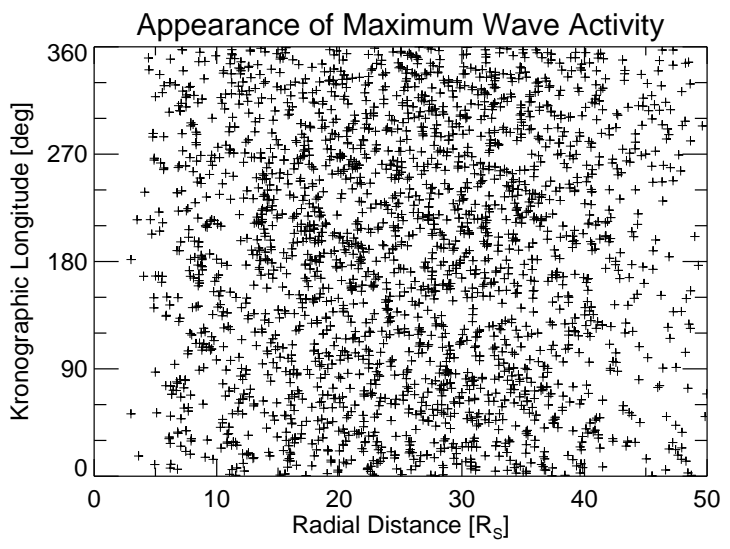

Fig. 13. Position of the maxima of the wave activity index in kronographic longitude over radial distance based on a variable rotation period.

is determined in this way. This value clearly deviates from the earlier determined and IAU-accepted value. Thus, the suggested model for a time-varying rotation rate is only applicable to the time interval covered by the Cassini mission so far.

Using the above derived apparent time-varying rotation rate the wave activity maxima and their relation to corrected Kronian longitude can be further discussed. Figure 13 displays the resulting distribution. It is as featureless as the uncorrected distribution.

\section{Summary and conclusion}

The results displayed in Fig. 4 suggest a possible relation between the large scale modulation of the Kronian magnetic field and the ULF wave activity. Such a relation could be due to a corotating magnetic field anomaly either caused by an internal non-axisymmetry of the Kronian dynamo or, for example, caused by a current system as proposed by Southwood and Kivelson (2007). Any such region of perturbation is locally causing a large-amplitude magnetic field disturbance decaying into shorter period oscillations such as suggested by Glassmeier (1995) for the Jovian magnetosphere. While the disturbance propagates through the magnetosphere only selected regions allow the excitation of ultra low frequency plasma waves. Some of the data intervals analyzed support this scenario. It would also imply that the ULF wave activity is confined to special regions in kronographic longitude related to the current longitude position of the exciting perturbation. The longitude distribution of the ULF wave activity (Fig. 9) which does not correspond to an accentuated longitude range implies, that the life-time of the waves in one of the selected regions is larger than the period of the exciting disturbance. Therefore we cannot determine which phase of 
the modulation excited the observed waves in an individual region of the Kronian magnetosphere.

Furthermore we seized the suggestion made by Giampieri et al. (2006) to correct the Kronian longitude system with respect to a new rotation rate and introduced a simple correction method based on the phase evolution of Saturn's prime meridian for two different rotation periods. The application of this method did not organize the longitude distribution of the ULF wave activity within distinguishable regions of preference, but it revealed a clear longitudinal drift of the maxima of the magnetic field modulation (Fig. 10) which suggests a time-variable period of the perturbation.

Therefore a time-variable kronographic longitude system was derived, based on the z-component of the magnetic field data, assuming a variable rotation period. The rotating system found well orders the background magnetic field modulation within an accentuated longitude range, hence indicating a suitable kronographic longitude system for the representation of the ULF wave activity. However, the application of this longitude system to represent the ULF wave activity information does not yield any systematic longitude distribution (Fig. 13). This questions our hypothesis.

Nevertheless, the simple model of an eccentric excitation and subsequent decay of a large amplitude perturbation needs more detailed considerations. A detailed model is required to take propagation effects within the magnetosphere, local conditions for the excitation of ULF waves in selected regions and considerations concerning the life-time of ULF waves in those special regions into account. Such considerations are beyond the scope of the present study.

Finally, we like to note that our time-variable Kronian longitude system is comparable to that one recently introduced by Kurth et al. (2007).

Acknowledgements. This work was financially supported by the German Bundesministerium für Wirtschaft und Technologie and the Deutsches Zentrum für Luft- und Raumfahrt under contract 50 OH 9901/4.

Topical Editor I. A. Daglis thanks two anonymous referees for their help in evaluating this paper.

\section{References}

Acton, C. H.: SPICE Products Available to the Planetary Science Community, in: Lunar and Planetary Institute Conference Abstracts, vol. 30 of Lunar and Planetary Institute Conference Abstracts, p. 1233, 1999.

Cecconi, B. and Zarka, P.: Model of a variable radio period for Saturn, J. Geophys. Res. (Space Physics), 110, 12203, doi:10. 1029/2005JA011085, 2005.

Cramm, R., Glassmeier, K.-H., Stellmacher, M., and Othmer, C.: Evidence for resonant mode coupling in Saturn's magnetosphere, J. Geophys. Res., 103, 11951-11960, doi:10.1029/98JA00629, 1998.
Desch, M. D. and Kaiser, M. L.: Voyager measurement of the rotation period of Saturn's magnetic field, Geophys. Res. Lett., 8, 253-256, 1981.

Dougherty, M. K., Kellock, S., Southwood, D. J., Balogh, A., Smith, E. J., Tsurutani, B. T., Gerlach, B., Glassmeier, K.-H., Gleim, F., Russell, C. T., Erdos, G., Neubauer, F. M., and Cowley, S. W. H.: The Cassini Magnetic Field Investigation, Space Sci. Rev., 114, 331-383, doi:10.1007/s11214-004-1432-2, 2004.

Espinosa, S. A. and Dougherty, M. K.: Periodic perturbations in Saturn's magnetic field, Geophys. Res. Lett., 27, 2785-2788, doi: 10.1029/2000GL000048, 2000.

Espinosa, S. A., Southwood, D. J., and Dougherty, M. K.: Reanalysis of Saturn's magnetospheric field data view of spin-periodic perturbations, J. Geophys. Res. (Space Physics), 108, 1085, doi: 10.1029/2001JA005083, 2003a.

Espinosa, S. A., Southwood, D. J., and Dougherty, M. K.: How can Saturn impose its rotation period in a noncorotating magnetosphere?, J. Geophys. Res. (Space Physics), 108, 1086, doi: 10.1029/2001JA005084, 2003b.

Espley, J. R., Cloutier, P. A., Brain, D. A., Crider, D. H., and Acuña, M. H.: Observations of low-frequency magnetic oscillations in the Martian magnetosheath, magnetic pileup region, and tail, J. Geophys. Res. (Space Physics), 109, 7213, doi: 10.1029/2003JA010193, 2004.

Fujita, S., Glassmeier, K. H., and Kamide, K.: MHD waves generated by the Kelvin-Helmholtz instability in a nonuniform magnetosphere, J. Geophys. Res., 101, 27317-27326, doi:10.1029/ 96JA02676, 1996.

Galopeau, P., Zarka, P., and Ortega-Molina, A.: Evidence of Saturn's magnetic field anomaly from Saturnian kilometric radiation high-frequency limit, J. Geophys. Res., 96, 14129-14140, 1991.

Giampieri, G., Dougherty, M. K., Smith, E. J., and Russell, C. T.: A regular period for Saturn's magnetic field that may track its internal rotation, Nature, 441, 62-64, doi:10.1038/nature04750, 2006.

Glassmeier, K.-H.: Magnetometer array observations of a giant pulsation event, J. Geophys. Zeitschrift Geophysik, 48, 127-138, 1980.

Glassmeier, K.-H.: Ultralow-frequency pulsations: Earth and Jupiter compared, Adv. Space Res., 16, 209, doi:10.1016/ 0273-1177(95)00232-4, 1995.

Glassmeier, K.-H. and Neubauer, F. M.: Low-frequency electromagnetic plasma waves at comet P/Grigg-Skjellerup: Overview and spectral characteristics, J. Geophys. Res., 98, 20921, doi: 10.1029/93JA02583, 1993.

Glassmeier, K.-H., Neubauer, F. M., Ness, N. F., and Acuna, M. H.: Standing hydromagnetic waves in the Io plasma torus - Voyager 1 observations, J. Geophys. Res., 94, 15063-15076, doi:10.1029/ JA094iA11p15063, 1989.

Glassmeier, K.-H., Motschmann, U., Mazelle, C., Neubauer, F. M., Sauer, K., Fuselier, S. A., and Acuna, M. H.: Mirror modes and fast magnetoacoustic waves near the magnetic pileup boundary of comet P/Halley, J. Geophys. Res., 98, 20955, doi:10.1029/ 93JA02582, 1993.

Glassmeier, K.-H., Klimushkin, D., Othmer, C., and Mager, P.: ULF waves at Mercury: Earth, the giants, and their little brother compared, Adv. Space Res., 33, 1875-1883, doi:10. 1016/S0273-1177(04)00010-9, 2004.

Goldreich, P. and Farmer, A. J.: Spontaneous axisymmetry breaking 
of the external magnetic field at Saturn, J. Geophys. Res. (Space Physics), 112, 5225, doi:10.1029/2006JA012163, 2007.

Gurnett, D. A., Persoon, A. M., Kurth, W. S., Groene, J. B., Averkamp, T. F., Dougherty, M. K., and Southwood, D. J.: The Variable Rotation Period of the Inner Region of Saturn's Plasma Disk, Science, 316, 442, doi:10.1126/science.1138562, 2007.

Khurana, K. K. and Kivelson, M. G.: Ultralow frequency MHD waves in Jupiter's middle magnetosphere, J. Geophys. Res., 94, 5255-5263, 1989.

Kurth, W. S., Lecacheux, A., Averkamp, T. F., Groene, J. B., and Gurnett, D. A.: A Saturnian longitude system based on a variable kilometric radiation period, Geophys. Res. Lett., 34, 2201, doi: doi:10.1029/2006GL028336, 2007.

Luhmann, J. G., Tatrallyay, M., Russell, C. T., and Winterhalter, D.: Magnetic field fluctuations in the Venus magnetosheath, Geophys. Res. Lett., 10, 655-658, doi:10.1029/GL010i008p00655, 1983.

Mathie, R. A. and Mann, I. R.: Observations of Pc5 field line resonance azimuthal phase speeds: A diagnostic of their excitation mechanism, J. Geophys. Res., 105, 10713-10728, doi: 10.1029/1999JA000174, 2000.

Seidelmann, P. K., Abalakin, V. K., Bursa, M., Davies, M. E., de Bergh, C., Lieske, J. H., Oberst, J., Simon, J. L., Standish, E. M., Stooke, P., and Thomas, P. C.: Report of the IAU/IAG Working Group on Cartographic Coordinates and Rotational Elements of the Planets and Satellites: 2000, Celestial Mechanics and Dynamical Astronomy, 82, 83-111, 2002.
Southwood, D. J.: Some features of field line resonances in the magnetosphere, Planet. Space Sci., 22, 483-491, doi:10.1016/ 0032-0633(74)90078-6, 1974.

Southwood, D. J.: A general approach to low-frequency instability in the ring current plasma, J. Geophys. Res., 81, 3340-3348, doi: 10.1029/JA081i019p03340, 1976.

Southwood, D. J. and Hughes, W. J.: Theory of hydromagnetic waves in the magnetosphere, Space Sci. Rev., 35, 301-366, 1983.

Southwood, D. J. and Kivelson, M. G.: Saturnian magnetospheric dynamics: Elucidation of a camshaft model, J. Geophys. Res. (Space Physics), 112, 12222, doi:10.1029/2007JA012254, 2007.

Tamao, T.: Transmission and coupling resonance of hydromagnetic disturbances in the non-uniform earth's magnetosphere, Sci. Rep. Tohoku Univ. Ser. 5, 17(2), 43, doi:10.1016/0032-0633(78) 90054-5, 1965.

Tsurutani, B. T. and Smith, E. J.: Hydromagnetic waves and instabilities associated with cometary ion pickup - ICE observations, Geophys. Res. Lett., 13, 263-266, 1986. 